\title{
The mitochondrion as a Swiss army knife: implications for cardiovascular disease
}

\author{
Stephen L. Archer
}

Received: 30 July 2010 / Accepted: 30 July 2010 / Published online: 14 August 2010

(C) Springer-Verlag 2010

Mitochondria are misunderstood...or perhaps, it is fairer to say that the diversity of their function is underappreciated. While the metabolic capacity of mitochondria to generate ATP is essential to mammalian life, it is increasingly clear that mitochondria have a repertoire of function worthy of an autonomous organism. To use a culinary analogy, students are taught that a mitochondrion functions as if it were a table knife - for cutting only; in fact, mitochondria are more aptly viewed as a Swiss army knife, controlling energetics, rates of cell proliferation, and apoptosis, and even the age and regenerative potential of cells. Dynamic probes that allow real-time imaging of mitochondria structure and function (photo-activated mitochondrial green fluorescent proteins), membrane potential (JC-1), and redox state (redox-sensitive mitochondrial-GFP) have enriched our understanding of mitochondrial biology. Mitochondria move, mitochondria fuse, mitochondria have their own membrane potential, and mitochondria generate their own redox environment. A few minutes watching these organelles move, divide, and join leaves even an experienced scientist excited, full of questions, and wonder at the undiscovered roles of these organelles that may once have been autonomous endosymbionts.

In this special edition of JMM, we examine the impact of some of the less appreciated aspects of mitochondrial function to cardiovascular diseases. Mitochondrial contents liaise through dynamic networks. Mitochondria communicate with the plasma membrane proteins, such as ion channels, through the production of reactive oxygen species

S. L. Archer $(\bowtie)$

Section of Cardiology, Department of Medicine,

University of Chicago,

5841 South Maryland Avenue, (MC6080),

Chicago, IL 60637, USA

e-mail: sarcher@medicine.bsd.uchicago.edu
(ROS). Through redox regulation of potassium and calcium channels, they can control the cell's ionic milieu and, hence, the proliferation/apoptosis balance. Links between mitochondrial function and transcription factors, such as HIF-1 $\alpha$, Myc, and NFAT, tie mitochondrial metabolism to cardiomyocyte function and vascular smooth muscle cell (VSMC) proliferation. Mitochondrial network dynamics and metabolism not only are crucial to normal cellular functions (such as maturation and contractility) but also play a pathophysiologic role in a host of cardiovascular diseases including the cardiometabolic syndrome, myocardial ischemia, pulmonary arterial hypertension $(\mathrm{PAH})$, and right ventricular hypertrophy $(\mathrm{RVH})$ and failure. It appears that vascular aging and stem-cell maturation also have a mitochondrial component.

To begin this eight-part special issue of the Journal of Molecular Medicine, Michael Frohman provides an overview of how the newly recognized mitochondrial capabilities have relevance for cardiovascular disease. Mitochondria exist not as isolated organelles but in dynamic networks. The balance between two actively regulated processes, fission (joining of mitochondria) and fusion (separation into discrete mitochondria), is an emerging area of research that has relevance to understanding disordered cell proliferation and apoptosis. Network integrity likely has effects on redox state and the integrity of the mitochondrial genome. Moreover, the mitochondrial reticulum is connected to and communicates with the endoplasmic reticulum, offering an intriguing area of research that has implications for calcium homeostasis. In addition, the biogenesis and degradation of mitochondria are almost certainly important in cardiovascular disease. Biogenesis (creation of new mitochondria) occurs in response to energy demands and thus is linked to metabolism through key fuel gauges, such as AMPK. The role of mitochondrial 
membrane potential in controlling the removal of damaged mitochondria through mitophagy (which can be viewed as an organellar equivalent to apoptosis) is an intriguing mechanism for quality control, which has already been implicated in certain cardiovascular diseases, including PAH.

Kane and Youle review the molecular mechanisms of mitochondrial fission and fusion, which occur under the guidance of fusogenic (e.g., the mitofusins and Opa1) and fissogenic (e.g., dynamin-related protein, Drp-1) proteins. One theory for the role of this networking relates to its ability to counterbalance the oxidative stress that occurs as mitochondria consume oxygen during oxidative phosphorylation. The resulting ROS are toxic to many components of the mitochondria, including mitochondrial DNA. The admixture of mitochondrial contents through fusion likely helps maintain and repair DNA, lipids, and proteins, thus preserving mitochondrial health. If network dynamics are impaired, oxidized proteins accumulate. Once mitochondrial damage is excessive (as indicated by membrane potential), they may be marked for degradation by mitophagy and excluded from participation in the network.

The arrangement of mitochondria within the cardiovascular system is contextual-varying between cell types. In VSMC, mitochondria permeate the cytosol-like electrical wiring in a house. In contrast, mitochondria in cardiac myocytes are neatly arranged in one of two compartments, either between the myofibrils or between the myofibrils and the cell membrane. The role of fission and fusion in the heart is largely terra incognita, although there is early evidence that loss of the fusogenic protein, Opal, is associated with a fragmented mitochondrial phenotype in heart cells following ischemia or in cardiomyopathy.

There is tremendous interest in harvesting the potential of endogenous or autologous stem cells to treat various cardiovascular diseases. Jalees Rehman offers an intriguing glimpse of mitochondria in human embryonic stem cells, suggesting that stem cells preserve their stemness by preserving a glycolytic mitochondrial-metabolic phenotype. It appears that undifferentiated, pluripotent stem cells have lower mitochondrial function and depressed rates of oxidative phosphorylation than do terminally differentiated cells. They rely instead on the preferential use of glycolysis as a major source of energy. He presents the concept that suppressed mitochondrial oxidative metabolism is both an indicator and determinant of the undifferentiated state of stem cells, relevant to pluripotency and self-renewal of stem cells. The hypothesized mitochondrial regulation of stem cell fate might allow metabolic programming of stem cells.

Next, we turn to Zheng and Xiao who summarize the emerging literature indicating that impaired expression or function of mitochondrial fusion proteins contributes to cardiovascular disease by modulating oxidative stress. Intriguingly, this group, upon cloning mitofusin-2 from rat
VSMCs, named it hyperplasia suppressor gene for its ability to suppress cell proliferation. The story continues to evolve, and it now appears that mitofusin-2's antiproliferative effects may occur, at least in part, independently of its role in mitochondrial fusion. Mitofusin- 2 may have therapeutic relevance as a means of suppressing restenosis. There are also therapeutic implications of fission. Emerging data suggest that inhibiting Drp-1 in the heart may protect the myocardium from ischemia.

Jun Ren et al. discuss the role that impaired myocardial mitochondrial biogenesis, fatty acid metabolism, and antioxidant defense mechanisms play in the metabolic syndrome, the triad of obesity, hypertension, and insulin resistance which is the new cardiovascular epidemic. They discuss the role of impaired myocardial mitochondrial biogenesis and diminished cardiac substrate flexibility and energetic efficiency in causing lipid accumulation and diastolic dysfunction in the heart. They review links between enhanced activity of the rennin-angiotensin-aldosterone system, ROS production, and mitochondrial-induced apoptosis. They also note a pathological positive feedback pathway between impaired insulin signaling, glucose metabolism, and mitochondrial dysfunction. Finally, the hypothesis is presented that a maladaptive, PGC- $1 \alpha$-mediated increase in mitochondrial biogenesis, occurring in response to energy deficiency, contributes to cardiac dysfunction in metabolic syndrome.

Peter Dromperis et al. present the link between mitochondrial metabolism and ROS production and the activity of transcription factors as an explanation for the increased proliferation/apoptosis balance in PAH. In PAH, SMC mitochondria have decreased pyruvate influx and decreased activity of Krebs' cycle. Moreover, there is hyperpolarization of the mitochondrial membrane potential. These hyperpolarized mitochondria behave as if they were hypoxic, making fewer ROS and creating a milieu that favors resistance to apoptosis. Suppression of mitochondrial ROS, below physiologic levels, leads to inhibition and downregulation of redox-sensitive potassium channels, resulting in accumulation of intracellular $\mathrm{K}^{+}$and $\mathrm{Ca}^{2+}$, with a resulting increase in proliferation and contraction, activation of transcription factors, such as NFAT, and suppression of apoptosis. The authors note remarkable similarities between PAH and cancer, the prototypic disorder of excess cell proliferation and impaired apoptosis. This article would please Otto Warburg, who described cancer's reliance on glycolysis (despite abundant oxygen) as a means of promoting pathologic cell proliferation. This has therapeutic implications, as demonstrated by the efficacy of pyruvate dehydrogenase kinase (PDK) inhibitors in PAH.

Piao et al. present the concept that in RVH, there is a reversible inactivation of mitochondrial glucose metabolism that leads to state of RV hibernation. In RVH, a vicious cycle of ischemia (whether related to reduced coronary perfusion pressure or microvascular disease) and transcrip- 
tion factor activation (e.g., HIF-1 $\alpha$ and Myc) creates a maladaptive dependence on glycolysis that limits contractility, through energy deprivation. They suggest that activating glucose oxidation can enhance RV function. First discussed is a direct mechanism to achieve this end, using dichloroacetate to inhibit PDK. Piao et al. also suggest the therapeutic strategy of enhancing glucose oxidation indirectly by activating Randle's cycle. Drugs that inhibit fatty acid oxidation, such as trimetazidine and ranolazine, can activate glucose oxidation and increase RV function. The concept of a maladaptive glycolytic shift leading a hibernating RV in RVH suggests mitochondrial pyruvate dehydrogenase complex as a new therapeutic target in RV failure.

Finally, Zoltan Ungvari et al. explore mitochondria and vascular aging. They review the links between mitochondrial oxidative stress and chronic low-grade vascular inflammation. This oxidant stress can lead to increased rates of endothelial apoptosis and development of vascular diseases in the elderly. Perhaps, we are not as "young as we feel"; rather, we are as young as our mitochondrial function. They explore the concept that evolutionarily conserved pathways (such as SIRT, Nrf2/ARE) control the aging process by linking cellular bioenergetics, mitochondrial function, and mitochondrial redox homeostasis to determine biological age. This raises the possibility that pharmacological or nutritional modulation of these pathways may delay aging-induced cardiovascular disease.

Sources of funding This work is supported by NIH-RO1-HL071115 and 1RC1HL099462-01, the American Heart Association (AHA) and the Roche Foundation for Anemia Research.

Disclosures None. 